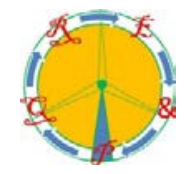

\title{
Energy harvesting approaches in IoT scenarios with very low ambient energy
}

\author{
A. Lopez-Martin, J.M. Algueta, I.R. Matías \\ Institute of Smart Cities \\ Public University of Navarra \\ Campus Arrosadia, 31006 Pamplona (Spain) \\ Phone number:+0034 948 169311, e-mail: antonio.lopez@unavarra.es
}

\begin{abstract}
The feasibility of multi-source energy harvesting in Internet of Things (IoT) scenarios with low and intermittent ambient energy is addressed. As a relevant case study, application to a smart cargo container system is analysed. The most relevant features of the main energy sources available in this target application are identified, and various transducers adapted to such sources are evaluated. Measurement results indicate that combined piezoelectric and thermoelectric generation inside cargo containers can significantly extend the battery lifetime of IoT end nodes embedded in such containers.
\end{abstract}

\section{Key words}

Energy Harvesting, Internet of Things, Wireless Sensor Networks, Multi-Source Harvesting, Hybrid Harvesting.

\section{Introduction}

The ongoing growth of world population and the simultaneous increase of development levels on a global basis have augmented energy demands notably. Although renewable energy sources are becoming more relevant, dependence on non-renewable energy sources is still evident and is having a clear environmental impact. This situation has forced governments and international organizations to promote politics trying to reduce energy consumption and to achieve a sustainable development model less aggressive to the environment. In this context, Information and Communication Technologies should play a key role to alleviate the problem by supporting more efficient energy management, but today, they are rather considered part of the problem. The impact of these technologies in terms of energy consumption is often not considered as they are very often "hidden" from the observer (buried cables, non-accessible rooms of computer servers, etc.). But in fact transmission and processing of information has a significant impact in terms of energy consumption. Proper design of these technologies would benefit to a large extent on the goal of efficient use of energy.
In this framework, energy efficiency is a critical aspect in the deployment of the Internet of Things (IoT) paradigm [1]. The disruptive nature of IoT is evidenced from estimations that envisage more than 50 billion connected devices by 2025. However, the vision of these IoT environments, which evolve around the idea of pervasive computing and connectivity anywhere, anytime and to any service, is nowadays still a utopia. Several envisaged IoT scenarios, such as those implying distributed monitoring of large areas or infrastructures, require myriads of wireless microsensor nodes forming ad hoc networks for the acquisition, processing and transmission of information. This vision is unfeasible both energetically and economically without highlyintegrated, ultra-low power end nodes. Moreover, currently these nodes often operate with batteries. Besides limiting the size reduction of these nodes, the large number of batteries to be used (and subsequently replaced and recycled) in these networks requiring massive deployment of nodes imply high maintenance costs and poses an additional thread to the environment.

Fortunately, the advent of energy harvesting techniques has improved this situation [2]. These techniques take advantage of the renewable energy available in the vicinity of these nodes (light, thermal gradients, vibrations, radiofrequency waves, etc.) to extend the lifetime of the batteries (or even to avoid these batteries), leading to energy-autonomous end nodes that can promote the IoT growth in a sustainable manner.

However, there are several scenarios of high practical interest (including most indoor areas not permanently illuminated) where ambient energy is scarce and intermittent, so that the use of energy harvesting is difficult. In this paper we analyse strategies to develop IoT applications in these scenarios, employing hybrid multi-source energy harvesting techniques. The implementation of a smart freight container is described as a practical case study. 


\section{Hybrid Energy Harvesting}

During the last years, several proposals of power management units for harvesting energy from a single transducer have been reported. The most common sources of energy are light [3], mechanical vibrations [4], thermal gradients [5] and radio frequency (RF) waves [6]. However, these techniques are restricted to applications where a relatively constant supply of the energy source employed is granted.

As mentioned above, scenarios without a stable energy harvesting source able to provide energy to the IoT node are common in practice. In this case, the most efficient approach is the use of hybrid, multisource energy harvesting systems, allowing to get energy from various heterogeneous sources in an opportunistic manner, depending on their availability. Most of the proposals to date concerning hybrid energy harvesting systems use a combination of light and vibrations as a robust and relatively cheap solution. In scenarios with thermal gradients sufficiently large and stable, thermoelectric generation is added to the energy mix. Thus, commonly employed transducers include photovoltaic cells, piezoelectric transducers and Peltier cells.

A simple approach to obtain a multi-source energy harvesting system is using a power OR'ing scheme [7], which is used in some commercial products such as the LTC3331 from Linear Technology and the MB39C811 from Cypress Semiconductor. It is a flexible and modular approach that allows including an arbitrary number of harvesting sources connected in parallel using diodes. Moreover, each source can implement an independent maximum power point tracking (MPPT) subsystem. However, the voltage drop on the forward diodes leads to power losses, and the size and cost are increased due to the need for an independent MPPT circuit for each source.

Instead of using a dedicated MPPT unit for each transducer, it is more efficient in terms of size and cost using a MIMO (multiple input, multiple output) converter, that can adapt the load to the transducer employed to maximize power efficiency [8]-[9] and to apply the resulting voltage to different energy storage elements such as batteries and supercapacitors. Based on this technique, the approach employed in this work is schematically depicted in Fig. 1.

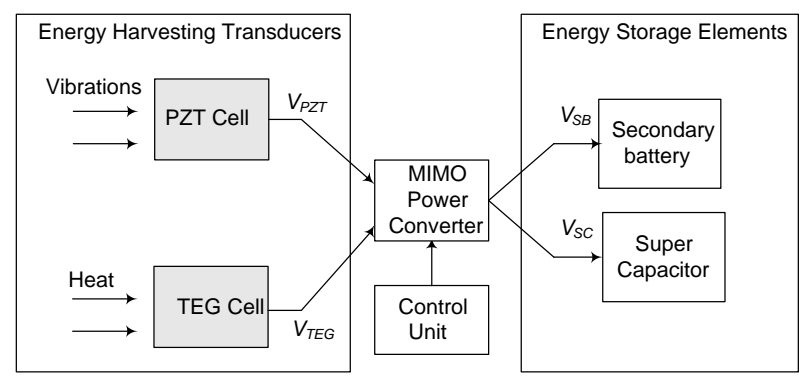

Fig. 1. Hybrid energy harvesting system.

\section{Case Study: Smart Container}

A key market segment for IoT applications is freight transport. Historically, it has experienced a steady growth and unbalanced use of different transport modes, with an increased relevance of truck transportation. Such scenario leads to inefficiency, higher costs and more environmental impact. The application of IoT technologies is envisaged as a key tool to change this scenario, providing intelligence to the transport systems and allowing them to be more resource-efficient, environmentally-friendly, safe and secure. A critical issue in this sense is transport of large containers that employ one of more modes of transportation (truck, rail or ship) [10]-[12].

\section{A. System Description}

Fig. 2 shows schematically the basic concept of a smart container system. The parameters sensed inside the container are transmitted wirelessly to the external node, which sends them to a Unified Logistic Information System (ULIS) to be used by the relevant stakeholders of the logistic chain. Current approaches [13]-[17] are often complex and costly (usually based on proprietary systems with satellite connectivity in each container), not fully exploiting the potential that these new technologies offer. Moreover, it is difficult to achieve autonomous sensing IoT nodes inside the container. The interior of a cargo container represents a very demanding scenario to perform energy harvesting, as available energy sources are weak and intermittent. Usually photovoltaic cells are discarded due to the unavailability of light inside the container when it is closed. The main energy source when the container is in motion comes from vibrations, although they show high variability depending on the characteristics of the vehicle transporting the container. For instance, in trucks on conventional roads, the obtained weighted RMS acceleration is between 0.4 and $1.2 \mathrm{~m} / \mathrm{s}^{2}$ with fundamental frequency $<100 \mathrm{~Hz}$ [18]. In maritime transport, vibration levels can vary widely depending on the characteristics of the ship, the cargo and the maritime zone where the displacement takes place. These values are usually high particularly for small ships sailing in open water. Railway transport shows generally the highest values of mean acceleration due to vibrations, with maximum values of about $20 \mathrm{~m} / \mathrm{s}^{2}$ and average levels of around $3 \mathrm{~m} / \mathrm{s}^{2}$, and fundamental frequencies of around $20-40 \mathrm{~Hz}$ [19]. Concerning thermal gradients, they may be significant but strongly depend on several factors affecting the container such as total or partial exposure to solar radiation, cooling and airflow systems [20]. Hence, the solution proposed by the authors in this scenario is the use of a multisource approach combining piezoelectric and thermoelectric transducers, as shown in Fig. 1. 


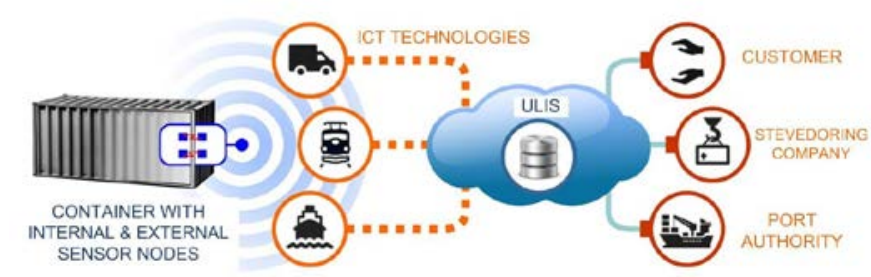

Fig. 2. Main concept of smart container system: Enabling technologies and their link with the main stakeholders of the logistic chain.

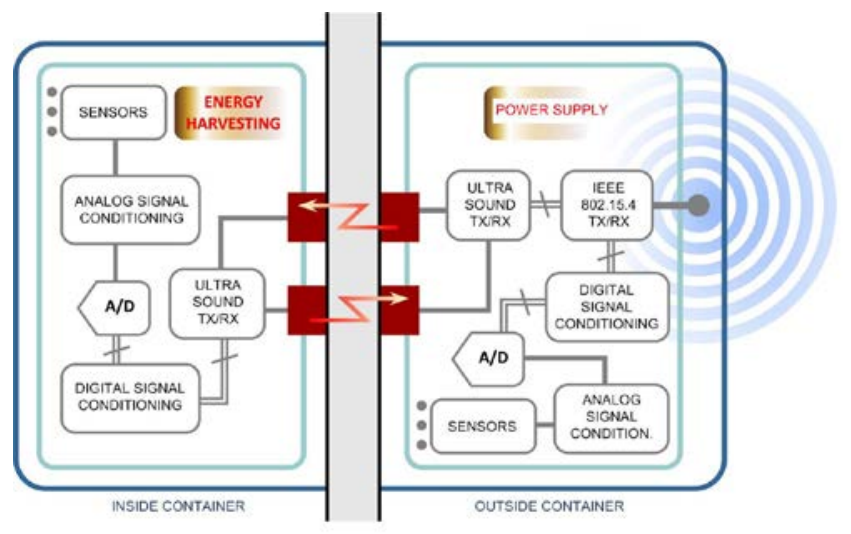

Fig. 3. Wireless sensor nodes inside and outside the container.

Figure 3 shows a simplified diagram of the internal and external nodes of the smart container developed [21]. The internal nodes sense environmental parameters of interest: motion, humidity, temperature, etc. These data are used to monitor the container, to preserve the optimal conditions for the load (such as maintaining the cold chain) and to detect intrusion. This information transmitted to an external node outside the container, which processes and transmits them to the coordinator node of the network. In order to avoid drilling the wall of the container to pass cables, communication between the internal and the external node through the metallic wall is made using ultrasound waves. A photograph of the first version of the internal node (still without energy harvesting transducers) is shown in Fig. 4, where the ultrasound transducer attached to the metallic wall can be seen. Internal container data are provided from temperature, humidity, motion and magnetic sensors. Analysis of the power savings achievable for this node by piezoelectric and thermoelectric energy harvesting is provided below.

\section{B. Piezoelectric Energy Harvesting}

Among the different transducers available to collect energy from vibrations, piezoelectric devices were chosen versus electromagnetic transducers due to their lower cost, simplicity, and suitability for the range of frequencies and accelerations typically attainable inside the container. Both piezoelectric cantilevers and diaphragms were analyzed although the operation frequency of commercially available diaphragms are usually beyond the frequency range in this scenario.

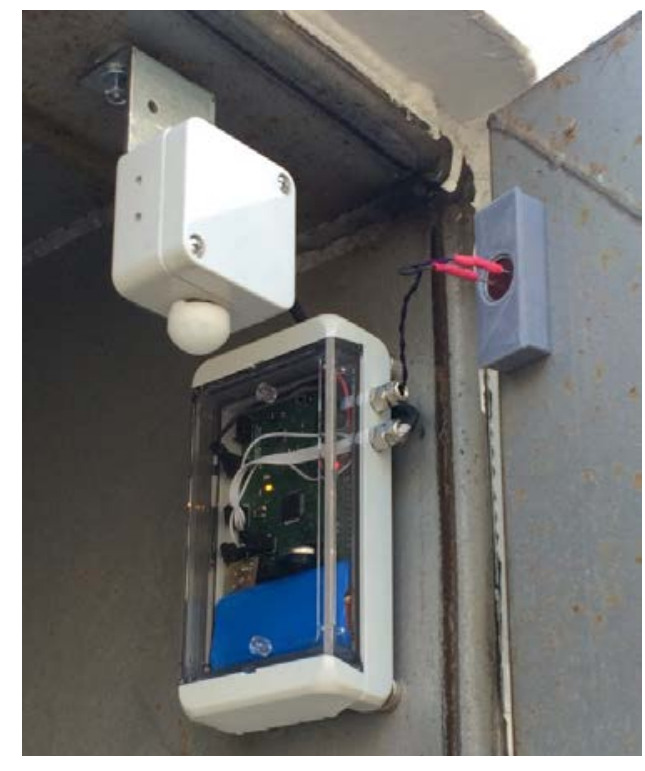

Fig. 4. Internal node of the container.

The experiments were carried out with a PASCO SF9324 actuator (shaker). The experimental setup is shown in Fig. 5. Figure 6 shows the measured output voltage achieved for two piezoelectric cantilevers of TE Connectivity Measurement Specialties as a function of the applied frequency, for a constant RMS acceleration of $100 \mathrm{mg}$. The load employed for measuring the output voltage was $1 \mathrm{M} \Omega$. Cantilever 1 corresponds to the 1005447-1 model, and Cantilever 2 is the 1005939 model. Both cantilevers include a tip mass that allows increasing sensitivity at the frequencies of interest for this application. According to the aforementioned data, the 1005447-1 model is more suitable for railway transportation, while both can be adequate for truck transportation.

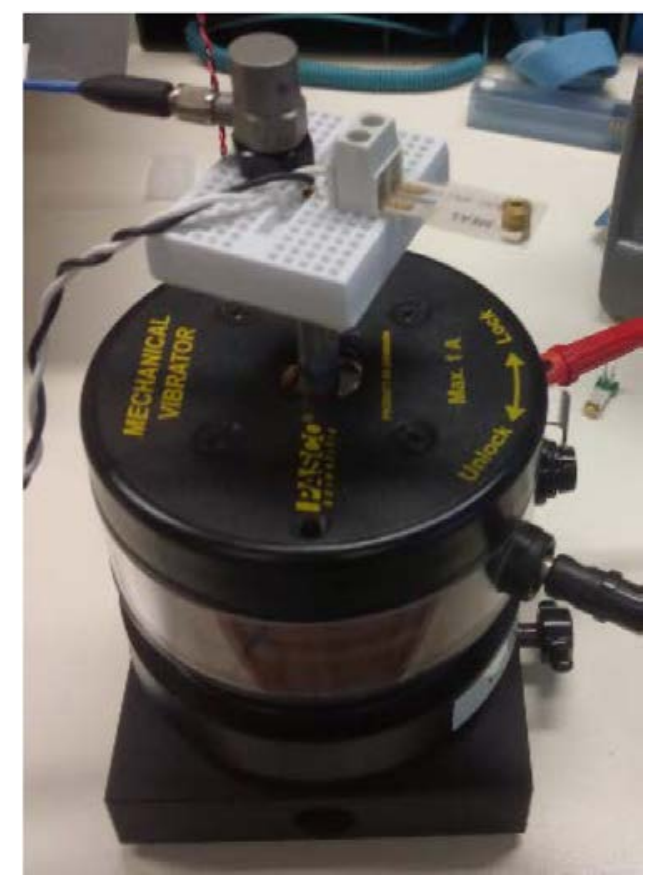

Fig. 5. Experimental setup for vibration harvesting. 


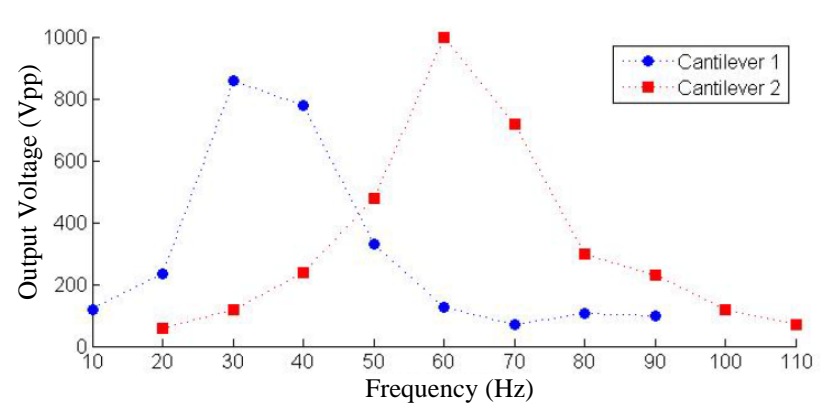

Fig. 6. Measured output voltage of two cantilevers.

\section{Thermoelectric Energy Harvesting}

In order to evaluate the feasibility of harvesting energy from thermal gradients inside the container, the experimental setup shown in Fig. 7 was employed. It includes a heater unit, a console, an aluminium plate, a support board with adjustable height and a thermocouple.

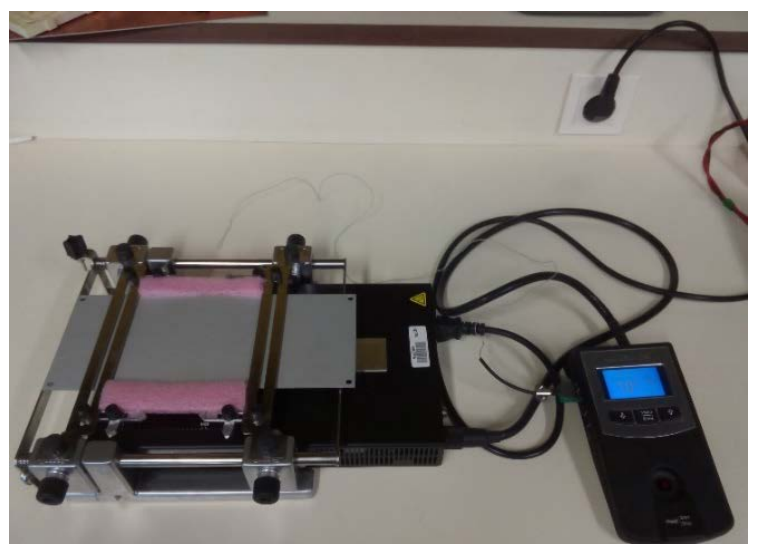

Fig. 7. Experimental setup for termoelectric harvesting.

A $40 \mathrm{~mm}$ x $40 \mathrm{~mm}$ thermoelectric generator (TEG) was attached to the aluminium plate, and a heat sink was placed at the other side. Due to the low TEG output voltage, the TEG output was processed by a LTC3108 boost converter. The maximum thermal gradient normally expected from the inner side of the contained wall and the interior of the container is $10{ }^{\circ} \mathrm{C}$, so we tested the available power in this situation. In order to control the thermal gradient, the TEG hot side temperature was sensed by the thermocouple and the cold side temperature was evaluated using a DEM100 infrared thermometer with laser. In this configuration for a thermal gradient of $10^{\circ} \mathrm{C}$ and a load of $25 \mathrm{k} \Omega$, a $3.3 \mathrm{~V}$ output is obtained, yielding an average power of $(3.3 \mathrm{~V})^{2} / 25 \mathrm{k} \Omega \approx 0.4 \mathrm{~mW}$.

\section{Communication through the Container Wall}

As mentioned above, the solution adopted for communication through the container wall was the use of ultrasound waves, in order to avoid drilling the container. The experimental setup used to test the communication system is shown in Fig. 8. A 2-mm thick steel plate emulates in the lab the container wall. Prowave EP250 ultrasound transducers were selected for transmission and reception, and a conventional charge amplifier was used to process the transducer output (using a TL08X opamp and a
$10 \mathrm{pF}$ integrating capacitor). A BPSK modulation was employed due to the low data rate required, with $41 \mathrm{kHz}$ carrier frequency. The acoustic continuity between the transducer and the metal wall is enforced using an epoxy gel. Both electrical and mechanical perturbations are applied to the steel plate using a signal generator and a shaker, respectively, in order to mimic the actual scenario of a cargo container. A FPGA board was used to generate the BPSK modulation and to evaluate the achieved Bit Error Rate (BER). Measurements show a BER $<2 \cdot 10^{-7}$ at 1.5 kbps even with moderate vibrations, evidencing the robustness of the system.

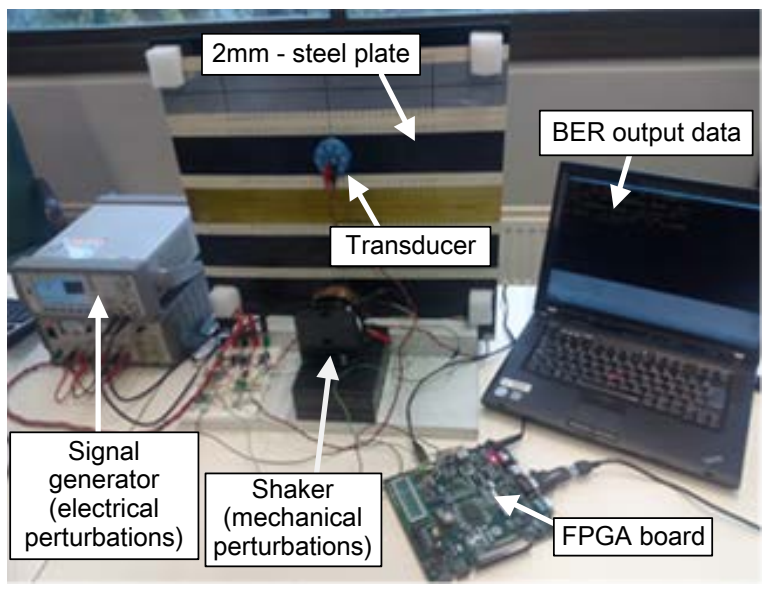

Fig. 8. Experimental setup employed for ultrasonic communications.

\section{Discussion and Conclusion}

The feasibility of energy harvesting techniques in IoT scenarios with low ambient energy available is discussed. A practical case study, corresponding to a smart cargo container, has been analysed in detail. The average power of the internal node of the container transmitting sensor data every 100 seconds is approximately $6 \mathrm{~mW}$. Considering it and the results measured for the piezoelectric and thermoelectric transducers, it can be concluded that they can be helpful to extend the battery life inside the container. The exact battery lifetime extension is difficult to calculate as it strongly depends on the context of the container (transportation means, intensity of insolation, etc.), but under adequate conditions it may be significant. For instance, according to the lab measurements in railway transportation with typical acceleration and with insolation providing a thermal gradient of $10^{\circ} \mathrm{C}$, it is estimated that battery life of the internal node could be doubled using an arrangement of five $40 \mathrm{~mm}$ x $40 \mathrm{~mm}$ TEG generator cells and a 1005447-1 cantilever.

Although not implemented in the current version of the system, an additional source of energy for the inner nodes can be ultrasonic waves coming from the external container node, using a similar setup as the one employed for communications. This solution could be helpful to provide extra energy when other harvesting sources are unavailable. 


\section{Acknowledgement}

This work was funded by Grant TEC2016-80396-C2-1-R (AEI/FEDER) and Obra Social La Caixa - Fundación Caja Navarra (Convocatoria 2018 de Captación de Talento).

\section{References}

[1] J.A. Stankovic, "Directions for the Internet of Things," IEEE IoT Journal, vol. 1, pp. 3-9, 2014.

[2] A. Lopez-Martin et al., "Energy harvesting microsystems based on the QFG MOS transistors", in Proc. IEEE EEEIC 2015.

[3] N. Khosro Pour, F. Krummenacher, M. Kayal, "Fully integrated solar energy harvester and sensor interface circuits for energy-efficient wireless sensing applications", J. Low Power Electron. Appl., vol. 3, no. 1, pp. 9-26, 2013.

[4] Y.K. Ramadass, A.P. Chandrakasan, "An efficient piezoelectric energy harvesting interface circuit using a biasflip rectifier and shared inductor", IEEE J. Solid-State Circuits, vol. 45, no. 1, pp. 189-204, 2010.

[5] D. Rozgic, D. Markovic, “A miniaturized $0.78-\mathrm{mW} / \mathrm{cm}^{2}$ autonomous thermoelectric energy-harvesting platform for biomedical sensors" IEEE Trans. Biomed. Circuit Syst., vol. 11, no. 4, pp. 773-783, 2017.

[6] A. Bertacchini, L. Larcher, M. Maini, L. Vincetti, S. Scorcioni, "Reconfigurable RF energy harvester with customized differential PCB antenna", J. Low Power Electron. Appl., vol. 5, no. 4, pp. 257-273, 2015.

[7] Y.K. Tan, S.K. Panda, "Energy harvesting from hybrid indoor ambient light and thermal energy sources for enhanced performance of wireless sensor nodes", IEEE Trans. Ind. Electron., vol. 58, no. 9, pp., 4424-4435, 2011.

[8] C. Park and P. H. Chou, "Power utility maximization for multiple supply systems by a load-matching switch," Proc. ISLPED, 2004, pp. 168-173.

[9] H. Shao, C. Y. Tsui, and W. H. Ki, "A single inductor dual output DC-DC converter with hybrid supplies for solar energy harvesting applications,” in Proc. ISLPED, 2009, pp. 69-74.

[10] T. Notteboom, "Containers shipping and ports: An overview," Review of Network Economics, vol. 3, pp. 86106, 2004

[11] T. Notteboom, "Strategic challenges to container ports in a changing market environment," Research in Transportation Economics, vol. 17, pp. 29-52, 2007.

[12] J. Muñuzuri, P. Cortés, J. Guadix and L. Onieva, "Modelling peak-hour urban freight movements with limited data availability," Computers and Industrial Engineering, vol. 59, pp. 34-44, 2010.

[13] J. Carn, "Smart container management: Creating value from real-time container security device data,” in Proc. IEEE Int Conf. Technol. Homel. Secur. (HST), 2011, pp. 457-465.

[14] W. Lang, R. Jedermann, D. Mrugala, A. Jabbari, B. KriegBrückner, and K. Schill, "The 'intelligent container' - A cognitive sensor network for transport management," IEEE Sens. J., vol. 11, no. 3, pp. 688-698, 2011.

[15] R. Jedermann, M. Nicometo, I. Uysal, and W. Lang, "Reducing food losses by intelligent food logistics," Philos. Trans. A. Math. Phys. Eng. Sci., vol. 372, no. 2017, p. 20130302, Jun. 2014.
[16] R. Jedermann, J. Palafox-Albarran, A. Jabarri, and W. Lang, Autonomous Cooperation and Control in Logistics. Elsevier, 2011, pp. 207-228.

[17] R. J. Katulski and A. Prof, "Self-organizing wireless monitoring system for cargo containers," Polish Maritime Research, vol. 16, no. 3, pp. 45-50, 2009.

[18] A. P. Cann, A. W. Salmoni, and T. R. Eger, "Predictors of whole-body vibration exposure experienced by highway transport truck operators," Ergonomics, vol. 47, no. 13, pp. 1432-1453, 2004.

[19] S. Bradai, S. Naifar, C. Viehweger, O. Kanoun, "Electromagnetic vibration energy harvesting for railway Applications", in Proc. International Conference on Engineering Vibration (ICoEV), 2017.

[20] J. Rodriguez-Bermejo, P. Barreiro, J.I. Robla, L. RuizGarcia, "Thermal study of a transport container" Journal of Food Engineering, vol. 80, no. 2, pp. 517-527. May 2007.

[21] A. Lopez-Martin, R.G. Carvajal and P. Cortés, "Smart ecosystem for a sustainable, safe and integrated freight transport”, in Proc. IEEE Emergitech, 2016. 